\title{
Cambios de hábitos alimentarios de los estudiantes de odontología de la Fundación Universitaria San Martín de Bogotá, Colombia
}

\author{
Edgar lbáñez ${ }^{1}$, Yeily Thomas ${ }^{2}$, Angela Bicenty ${ }^{3}$, Judith Barrera ${ }^{4}$, \\ Jeannette Martínez ${ }^{5}$, Ruby Gerena ${ }^{6}$.
}

1. Ingeniero U. Distrital, Especialista en Estadística U. Nacional, Especialista en Docencia Universitaria U. El Bosque, Candidato a la maestría de Epidemiologia Clínica U. Nacional, Docente de Investigación Universidad San Martín y Universidad el Bosque.

2. Odontóloga del Colegio Odontológico, Especialista en Epidemiología, Universidad el Rosario-CES, Docente de Investigación Universidad San Martín.

3. Odontóloga U. San Martín, Especialista en Gerencia Social de la Educación U. Pedagógica Nacional, Docente de Investigación y Pre clínica Universidad San Martín.

4.Odontóloga U El Bosque, Especialista en Epidemiología, Universidad del Rosario-CES, Especialista en Docencia Universitaria U El Bosque, Docente de Investigación Universidad San Martín.

5. Licenciada en educación especial, Especialista en dificultades en aprendizaje escolar, Magíster en educación y candidata en doctorado en pedagogía social.

6. Psicóloga Universidad Nacional, Especialista en Aprendizaje Autónoma UNAD.

Correspondencia: edgant111@yahoo.com

Recibido 21-04-08 / Aceptado 16-05-08

\section{Resumen}

En este artículo se muestran los resultados de una investigación cuyo objetivo fue determinar y describir los hábitos alimentarios de los estudiantes de odontología de la Fundación Universitaria San Martín de Bogotá, Colombia. El tipo de estudio fue observacional de corte transversal en una población de universitarios de primero a décimo semestre. La muestra fue de 319 estudiantes, como criterio de inclusión se tomó el estar matriculados al primer semestre de 2008. Las variables involucradas en el estudio fueron, hábitos alimentarios, semestre, género, edad, número de comidas ingeridas durante el día, clase de alimentos que consume, tiempo dedicado a comer, dinero destinado para alimentarse y criterios para elegir un alimento.

La edad promedio fue de 21,3 33,7 años, en donde el Índice de Masa Corporal se clasificó en bajo peso el | I\% ( $n=35)$, normal el 76,8\% ( $n=245)$, sobrepeso el 9, I\% ( $n=29)$ y obesidad I,3\% ( $n=6)$. Se encontraron diferencias significativas entre 3 a 4 comidas diarias (prueba t, $p=0.03$ ) antes y después de ingresar a la universidad siendo actualmente un $42 \%(n=134)$ y antes un $52,7 \%(n=168)$. El motivo predominante de consumir alguna comida menos de tres veces a la semana fue la falta de tiempo. Los alimentos que más se consumen son: lácteos al desayuno en un $64,3 \%(n=142)$, carnes, huevos y leguminosas a la hora del almuerzo y cena en un $77,2 \%$ $(n=186)$ y $58,5 \%(n=137)$ respectivamente. Se concluyó que el cambio en el estilo de vida de los estudiantes por ingresar a la universidad se refleja en los hábitos alimentarios, disminuyendo el número de comidas diarias por motivos como la falta de tiempo y dificultades económicas.

Key Words: food habits, body mass index, nutrition, overweight, quality of life. 


\section{Abstract \\ Changes of nourishing habits of the dentistry students of the University Foundation San Martin, Bogotá, Colombia}

This article shows the results of a research project whose objective was to determine and describe the nourishing habits among San Martin University dentistry students in Bogota, Colombia. The type of study was observational cross-section in a college student population from first to tenth semester. The sample consisted of 319 students. The inclusion criterion was to be registered in the first semester of 2008. The variables involved in this study were: nourishing habits, semester, gender, age, number of meals eaten during the day, class of food that they ate, time allotted to eat, money destined to buy food, and criteria to choose a food.

The average age was $21.3 \pm 3.7$ years, where the Body Mass Index was classified in low weight at I I \% ( $n=35)$, normal at 76.8\% $(n=245)$, overweight at $9.1 \%(n=29)$ and obesity at I. $3 \%(n=6)$. Relevant differences were found between 3 to 4 daily meals ( $t$ test, $p=0.0322$ ), before and after enrolling the university, being at present $42 \%(n=134)$ and before $52.7 \%(n=168)$. The predominant reason to consume some food fewer than three times a week was the lack of time. The foods that they eat more are: milk at breakfast $64.3 \%(n=142)$, meats, eggs, and vegetables at lunch and dinner in $77.2 \%(n=186)$ and $58.5 \%(n=137)$ respectively. It was concluded that changes in the student life style upon entering university were reflected in the nourishing habits, diminishing the number of daily meals by reasons such as lack of time and economic difficulties.

Palabras clave: calidad de vida, hábitos alimentarios, índice de masa corporal, nutrición, sobrepeso.

\section{Introducción}

El ingreso a la universidad genera una serie de cambios en la vida de los jóvenes que van desde separarse de su familia, en el caso de los estudiantes que tienen que desplazarse a las ciudades en busca de la universidad y dejar sus hogares; además de adaptarse a nuevas normas, compañeros de estudio, niveles elevados de estrés y manejo del tiempo. Dentro de estos cambios, la alimentación resulta uno de los factores menos visibles en los estudiantes, pero que generan mayores problemas en la salud a mediano y largo plazo. Una adecuada alimentación es un factor muy importante para la salud; en los universitarios se ha encontrado un déficit en la alimentación balanceada, ya sea por moda, la falta de tiempo libre o el escaso dinero disponible (1). Estudios demuestran cómo es más importante la imagen corporal que la nutrición, sin tener en cuenta el valor que posee cada tipo de alimento para la actividad humana (2). Durante los últimos años expertos en nutrición y salud han concluido que la calidad de vida de una persona puede mejorar si cambia sus hábitos alimentarios e incrementa su nivel de actividad física (3). La elección de los alimentos que se consumen diariamente es de gran importancia para mantener o perder peso. Los dos grupos que deben predominar en las comidas diarias son las verduras y las frutas. Se recomienda comer dos tazas de frutas y dos y media de verduras. Tampoco deben faltar los granos (3 onzas) y los productos lácteos (3 tazas) en la alimentación diaria (2).

La alimentación humana tiene dos dimensiones fundamentales: biológica y cultural. Es una forma de comunicación en la que se va de lo nutritivo a lo simbólico y de lo individual a lo colectivo. En este proceso se establecen prácticas alimentarias que están relacionadas directamente con aspectos como el económico, el acceso a los alimentos, los gustos a partir de las costumbres alimenticias por regiones, las horas de las comidas, los horarios y tiempos disponibles. Estos hábitos alimenticios están influenciados por el ambiente familiar social y cultural que determinan el modo en cómo se come y lo qué se come (4).

Debido a la diversidad de actividades de los estudiantes universitarios como asistir a clases, hacer trabajos en grupo, estudiar a solas, hacer sus prácticas en clínicas, entre 
otras, conducen a un desmejoramiento de su calidad de vida y a la adquisición de hábitos pocos saludables, como malos hábitos alimentarios, pues ellos deben optimizar su tiempo para cumplir con todos sus quehaceres cotidianos, lo que genera una demanda alta de estrés emocional y físico (5).

El objetivo de este estudio fue evaluar los cambios en los hábitos alimentarios en los estudiantes de odontología antes y después de ingresar a la universidad, determinar el índice de masa corporal e identificar los tipos de alimentos que consumen.

\section{Materiales y métodos}

El estudio fue observacional de corte transversal, en una población de estudiantes de la Fundación Universitaria San Martín, de Bogotá, Colombia. El tipo de muestreo fue estratificado por semestre por afijación proporcional y el tamaño de muestra fue de 319 estudiantes. Dentro de los criterios de inclusión se aceptaron estudiantes matriculados al primer semestre de 2008 y como criterios de exclusión el no contestar el instrumento o no encontrarse el día de la toma de datos. La variables involucradas en el estudio fueron hábitos alimentarios: número de comidas ingeridas durante el día, clase de alimentos que consume, tiempo dedicado a comer, dinero destinado para alimentarse y criterios para elegir un alimento. Se incluyeron datos de semestre, género, edad y lugar de procedencia.

Para el análisis de la clase de alimentos consumidos se utilizaron las Guías Alimentarias del Plan Nacional de Alimentación y Nutrición del Instituto Colombiano de Bienestar Familiar - ICBF. Las clasificaciones del índice de masa corporal según la Organización Mundial de la Salud - OMS en 1995 fueron: en bajo peso $(<18.5 \mathrm{Kg} /$ $\mathrm{m} 2$ ), normal (18.5 a $24.9 \mathrm{Kg} / \mathrm{m} 2$ ), sobrepeso (25 a 29.9 $\mathrm{Kg} / \mathrm{m} 2)$ y obesidad $(>30 \mathrm{Kg} / \mathrm{m} 2)$.

Se describieron las variables cualitativas mediante distribuciones de frecuencias y porcentuales, variables cuantitativas con medidas de tendencia central como el promedio y medidas de dispersión como la desviación estándar. Se midió la variación mediante el coeficiente de variación u homogeneidad (0-10\% homogéneo, 11-20\% medianamente homogéneo, mayor del 20\% heterogéneo). Para determinar diferencias se utilizo la distribución de t Student con una significancia del 5\%. Para el cálculo del tamaño de la muestra la confiabilidad fue del $95 \%$ y la potencia del $80 \%$. Se digitó y depuró en Excel versión 2003 y se proceso la información en el paquete Epi Info versión 3.4.3.

\section{Resultados}

\section{Características socio demográficas}

El género predominante fue el femenino con un 74.9\% ( $n=239)$. De Bogotá son el 51.7\% ( $n=165)$, de Cundinamarca el $6.6 \%(n=21)$ y de otros lugares de Colombia el 40.8\% ( $\mathrm{n}=130)$. La convivencia de los estudiantes con familiares fue del $80.1 \%(n=254)$, con amigos de $7.3 \%(n=23)$, solos en un $9.5 \%(n=30)$ y otros $3.2 \%(\mathrm{n}=10)$. La disponibilidad de recursos económicos propios y/o familiares con menos de un salario mínimo fue de $12.8 \%(\mathrm{n}=39)$, de 1 a 3 salarios mínimos en un $50 \%(\mathrm{n}=152)$, entre 4 y 5 salarios en un $29.3 \%(\mathrm{n}=89)$ y más de 6 salarios mínimos en un 7.9\% ( $\mathrm{n}=24)$.

La edad promedio fue de $21.3 \pm 3.7$ años, con un mínimo de 16 y un máximo de 45 años. La variabilidad fue medianamente homogénea $(\mathrm{CV}=17.4 \%)$. El peso promedio fue de $58.2 \pm 9.5 \mathrm{Kg}$ con un mínimo de $40 \mathrm{y}$ un máximo de $96 \mathrm{Kg}$, la variabilidad fue medianamente homogénea $(\mathrm{CV}=16.3 \%)$. El Índice de Masa Corporal (IMC) en bajo peso se encontró el 11\% ( $n=35)$, en peso normal el $76.8 \%(n=245)$, en sobrepeso el $9.1 \%(n=29)$ y obesidad $1.3 \%(n=6)$.

\section{Hábitos alimentarios}

Antes de ingresar a la universidad la frecuencia de número de comidas diarias fue de 1 a 2 en un $41.7 \%$ $(\mathrm{n}=133)$, de 3 a 4 comidas en un $52.7 \%(\mathrm{n}=168)$ y 5 o más en un $5.6 \%(\mathrm{n}=18)$. El número de comidas que actualmente se consume es de 1 a 2 en un $52.4 \%$ ( $n=167)$, de 3 a 4 comidas en un $42 \%$ ( $n=134)$ y 5 o más en un $5.6 \%$ $(\mathrm{n}=18)$. Comparando entre el número de comidas diarias consumidas antes y después de ingresar a la universidad se encontraron diferencias significativas entre 3 a 4 comidas (prueba t, $\mathrm{p}=0.03$ ) siendo menores actualmente.

En el desayuno se encuentran hábitos similares en cuanto a la costumbre de desayunar y en cuanto al número de veces por semana, antes y después de ingresar a la universidad. Los motivos más predominantes por 
Tabla 1. Distribución de hábitos alimentarios del desayuno, almuerzo y comida.

\begin{tabular}{|c|c|c|c|c|c|c|c|}
\hline & \multirow[b]{2}{*}{ Categorías } & \multicolumn{2}{|c|}{ Desayuno } & \multicolumn{2}{|c|}{ Almuerzo } & \multicolumn{2}{|c|}{ Comida } \\
\hline & & $\mathrm{N}$ & $\%$ & $N$ & $\%$ & $\mathrm{~N}$ & $\%$ \\
\hline $\begin{array}{l}\text { Acostumbraba a (desayunar), } \\
\text { (almorzar), (comer) } \\
\text { antes de ingresar a la } \\
\text { universidad }\end{array}$ & No & $\begin{array}{l}235 \\
83\end{array}$ & $\begin{array}{l}73.9 \\
26.1\end{array}$ & $\begin{array}{l}225 \\
89\end{array}$ & 71.7 & $\begin{array}{l}193 \\
102\end{array}$ & 65.4 \\
\hline $\begin{array}{l}\text { Número de veces a la semana } \\
\text { Que acostumbraba, }\end{array}$ & $\begin{array}{l}3 \text { o menos días } \\
\text { más de } 3 \text { días }\end{array}$ & \begin{tabular}{|l|}
16 \\
215
\end{tabular} & $\begin{array}{l}6.9 \\
93.1\end{array}$ & \begin{tabular}{|l|}
30 \\
192 \\
\end{tabular} & $\begin{array}{l}13.5 \\
86.5\end{array}$ & \begin{tabular}{|l|}
21 \\
174
\end{tabular} & $\begin{array}{l}10.8 \\
89.2\end{array}$ \\
\hline $\begin{array}{l}\text { Actualmente (desayuna), } \\
\text { (almuerza),(cena) }\end{array}$ & $\begin{array}{l}\mathrm{Si} \\
\text { No }\end{array}$ & $\begin{array}{l}225 \\
87\end{array}$ & $\begin{array}{l}72.1 \\
27.9 \\
\end{array}$ & $\begin{array}{l}246 \\
71 \\
\end{array}$ & $\begin{array}{l}77.6 \\
22.4 \\
\end{array}$ & $\begin{array}{l}237 \\
66 \\
\end{array}$ & $\begin{array}{l}78.2 \\
21.8\end{array}$ \\
\hline $\begin{array}{l}\text { Número de veces a la semana } \\
\text { Que acostumbra }\end{array}$ & $\begin{array}{l}3 \text { o menos días } \\
\text { más de } 3 \text { días }\end{array}$ & \begin{tabular}{|l|}
21 \\
197 \\
\end{tabular} & $\begin{array}{l}9.6 \\
90.4 \\
\end{array}$ & \begin{tabular}{|l|}
37 \\
205 \\
\end{tabular} & $\begin{array}{l}15.3 \\
84.7 \\
\end{array}$ & \begin{tabular}{|l|}
32 \\
198 \\
\end{tabular} & $\begin{array}{l}13.9 \\
86.1\end{array}$ \\
\hline $\begin{array}{l}\text { Motivos de (desayunar), } \\
\text { (almorzar), (cenar) menos } \\
\text { de } 3 \text { veces a la semana }\end{array}$ & $\begin{array}{l}\text { Dificultades económicas } \\
\text { Falta de tiempo } \\
\text { Por costumbre } \\
\text { Por dieta } \\
\text { Poca disponibilidad de } \\
\text { alimentos en la universidad }\end{array}$ & \begin{tabular}{|l}
0 \\
14 \\
3 \\
1 \\
2
\end{tabular} & $\begin{array}{l}0 \\
77.8 \\
16.7 \\
5.6 \\
\\
11.1\end{array}$ & $\begin{array}{l}7 \\
24 \\
6 \\
1 \\
2\end{array}$ & $\begin{array}{l}20.6 \\
70.6 \\
17.6 \\
2.9 \\
5.9\end{array}$ & $\begin{array}{l}6 \\
11 \\
7 \\
2 \\
0\end{array}$ & $\begin{array}{l}24.0 \\
44.0 \\
28.0 \\
8.0 \\
0.0\end{array}$ \\
\hline $\begin{array}{l}\text { Acostumbra a (desayunar), } \\
\text { (almorzar), (cenar) en: }\end{array}$ & $\begin{array}{l}\text { Casa } \\
\text { Cafetería } \\
\text { Restaurante } \\
\text { Trae de la casa } \\
\text { Otro }\end{array}$ & $\begin{array}{l}202 \\
24 \\
7 \\
5 \\
2\end{array}$ & $\begin{array}{l}92.7 \\
11.0 \\
3.2 \\
2.3 \\
0.9\end{array}$ & $\begin{array}{l}135 \\
108 \\
12 \\
17 \\
9\end{array}$ & $\begin{array}{l}55.8 \\
44.6 \\
5.0 \\
7.0 \\
3.7\end{array}$ & $\begin{array}{l}224 \\
2 \\
- \\
- \\
8\end{array}$ & $\begin{array}{l}97.8 \\
0.9 \\
- \\
- \\
3.5\end{array}$ \\
\hline Alimentos de consumo & $\begin{array}{l}\text { Cereales, tubérculos y } \\
\text { plátanos } \\
\text { Hortalizas y verduras } \\
\text { Frutas } \\
\text { Carnes, huevos, leguminosas } \\
\text { - } \\
\text { secas y mezclas vegetales } \\
\text { Lácteos } \\
\text { Grasas } \\
\text { Otros }\end{array}$ & $\begin{array}{l}82 \\
5 \\
68 \\
87 \\
\\
142 \\
16 \\
10\end{array}$ & $\begin{array}{l}37.1 \\
2.3 \\
30.8 \\
\\
39.4 \\
\\
64.3 \\
7.2 \\
4.5\end{array}$ & $\begin{array}{l}105 \\
71 \\
31 \\
186 \\
17 \\
58 \\
2\end{array}$ & $\begin{array}{l}43.6 \\
29.5 \\
12.9 \\
77.2 \\
\\
7.1 \\
24.1 \\
0.8\end{array}$ & $\begin{array}{l}73 \\
47 \\
45 \\
137 \\
\\
70 \\
42 \\
12\end{array}$ & $\begin{array}{l}31.2 \\
20.1 \\
19.2 \\
58.5\end{array}$ \\
\hline $\begin{array}{l}\text { Horario de desayuno(D) } \\
\text { Almuerzo(A) }\end{array}$ & $\begin{array}{l}\text { Regular } 7 \text { am a } 10 \text { am.(D) } \\
12 \mathrm{~m} \text { a } 2 \mathrm{pm}(\mathrm{A}) 6 \text { a } 8 \mathrm{pm}(\mathrm{C}) \\
\text { Irregular (otros horarios) }\end{array}$ & $\begin{array}{l}72 \\
150\end{array}$ & $\begin{array}{l}32.4 \\
67.6\end{array}$ & $\begin{array}{l}125 \\
115\end{array}$ & $\begin{array}{l}52.1 \\
47.9\end{array}$ & $\begin{array}{l}60 \\
176\end{array}$ & $\begin{array}{l}25.4 \\
74.6\end{array}$ \\
\hline
\end{tabular}

*D: Desayuno; A: Almuerzo; C: Comida

los cuales desayunan tres o menos veces a la semana son por falta de tiempo y por costumbre. Las personas acostumbran a desayunar en la casa y en la cafetería de la universidad. Los alimentos que más consumen son lácteos seguido de la carne, huevos, leguminosas secas y mezclas vegetales. El horario de desayuno más común es de 5 a 7 de la mańana.

En el almuerzo se encuentran hábitos similares en cuanto a la costumbre de almorzar y en cuanto al número de veces por semana, antes y después del ingreso a la universidad. Los motivos más predominantes por los cuales almuerzan tres o menos veces a la semana son por falta de tiempo y por dificultades económicas. Las personas acostumbran almorzar en la casa y en la cafetería de la universidad. Los alimentos que más consumen son la carne, huevos, leguminosas secas y mezclas vegetales seguido de cereales, tubérculos y plátanos. El horario de almuerzo más común es de $12 \mathrm{~m}$ a $2 \mathrm{pm}$.

En la comida se encuentran hábitos similares en cuanto a la costumbre de comer y en cuanto al número de veces por semana antes y después del ingreso a la universidad. Los motivos más predominantes por los cuales comen tres o menos veces a la semana son por falta de tiempo y por dificultades económicas. Las personas acostumbran a cenar 


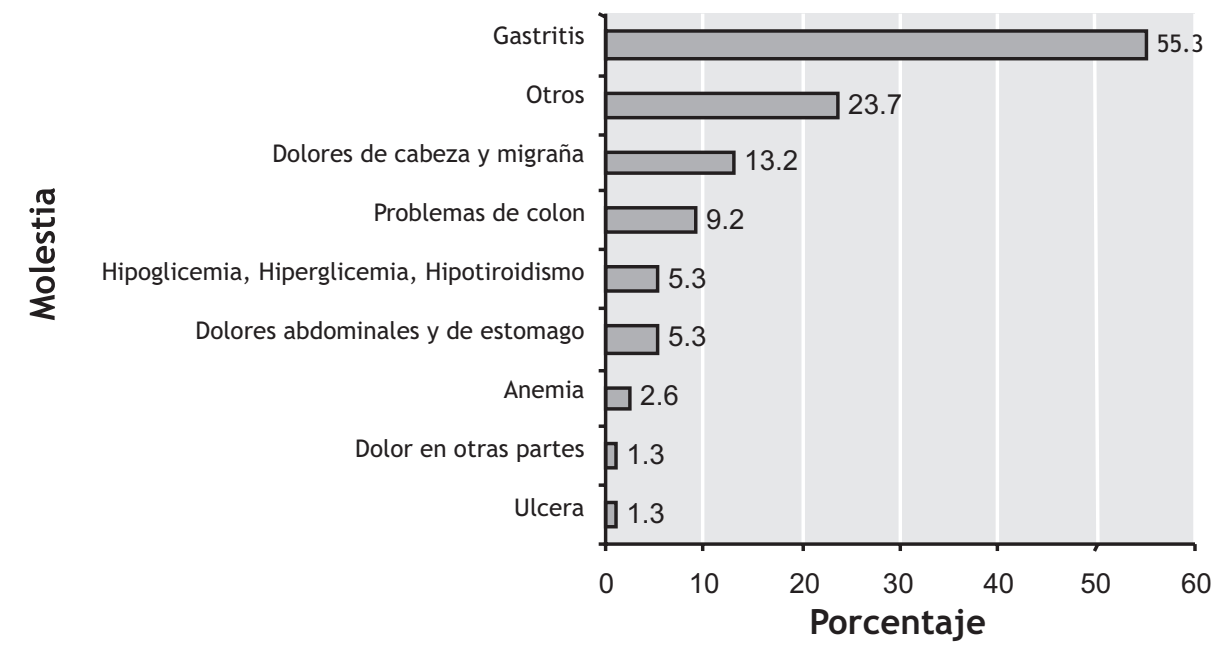

Grafica 1. Distribución de molestias de salud percibidas.

en la casa y en la cafetería de la universidad. Los alimentos que más consumen son la carne, huevos, leguminosas secas y mezclas vegetales seguido de cereales, tubérculos y plátanos. El horario de comida más común es de $8 \mathrm{pm}$ a $10 \mathrm{pm}$, Tabla 1.

Los refrigerios se acostumbraban a comer en el 68\% $(\mathrm{n}=208)$ de los estudiantes y actualmente el $68.5 \%$ $(\mathrm{n}=198)$. Con respecto al consumo por días en la semana, más de 3 días, antes el 75.3\% ( $\mathrm{n}=155)$ y ahora el 68\% $(\mathrm{n}=136)$. Los alimentos preferidos para los refrigerios fueron los lácteos en un 49.2\% ( $\mathrm{n}=94)$, seguido de las frutas en un $47.6 \%(n=91)$, grasas en un $19.9 \%(n=38)$, carnes y huevos en un $13.6 \%(\mathrm{n}=26)$, hortalizas y verduras en un $3.1 \%(n=6)$ y otros alimentos en un $11 \%(n=21)$.

\section{Percepción acerca de los hábitos alimentarios}

En el momento de elegir un alimento los estudiantes piensan en sus gustos en un $57.2 \%(n=179)$, en su costo en un $34.5 \%(n=108)$, en su calidad en un $31 \%(n=91)$, en su valor nutritivo en un $18.5 \%(\mathrm{n}=58)$, en recomendaciones médicas en un $5.1 \%(n=16)$, en las recomendaciones del nutricionista en un $4.5 \%(\mathrm{n}=14)$ y en otros motivos un $0.3 \%(\mathrm{n}=1)$. Han modificado sus hábitos alimentarios un 91.3\%( $\mathrm{n}=283)$, debido a las siguientes consideraciones: disponibilidad de tiempo en un $79.9 \%(\mathrm{n}=223)$, recursos económicos en un $22.6 \%(n=63)$, poca variedad en comidas en un $20.4 \%(n=57)$, dietas especiales en un
$7.2 \%(\mathrm{n}=20)$, problemas de salud en un $1.8 \%(\mathrm{n}=5)$ y otras consideraciones en un $2.9 \%(\mathrm{n}=8)$.

Desde el inicio de la carrera el estudiante ha percibido disminución del peso en un 50.2\% ( $\mathrm{n}=137)$, aumento del peso en un 20.9\% ( $n=57)$, se ha mantenido en su peso en un $28.9 \%(\mathrm{n}=79)$. La disminución del peso promedio fue de $5.4 \pm 3.3$ kilos con mínimo de 1 kilo y un máximo de 21 kilos, la variabilidad fue heterogénea $(\mathrm{CV}=61.7 \%)$. Con respecto al aumento el promedio fue de $5.9 \pm 3$ kilos, con un mínimo de 1 kilo y un máximo de 15 kilos, la variabilidad fue heterogénea $(\mathrm{CV}=50.6 \%)$. Las molestias de salud más comunes asociadas a los hábitos alimentarios fueron: gastritis, otros, dolor de cabeza y migraña, Grafica 1.

\section{Discusión}

Una nutrición sana es uno de los principales factores del estilo de vida y de la prevención de enfermedades crónicas en la adultez. Por su influencia en el desarrollo mental y físico de las personas, es un factor determinante de la capacidad para trabajar y de la expectativa de vida. Con respecto al IMC según los criterios de la OMS (6), se encontró que en bajo peso están el 11\% de los estudiantes, en peso normal el 76,8\%, y en sobrepeso el 9,1\%. Estudios comparativos muestran que en una población de estudiantes universitarios argentinos (1) los resultados fueron similares, con excepción del bajo peso en donde se reportó en $5.1 \%$. En estudiantes universitarios chinos (7) los valores de sobrepeso se presentan en un $2.5 \%$ y 
obesidad en un $0.4 \%$. En población japonesa el bajo peso es frecuente con un 24.7\% (8). En Estados Unidos el 35\% de los estudiantes en el año 2000 fueron reportados con sobrepeso u obesidad (9).

En el estudio de Kolarzyk y colaboradores (2003) en Cracovia (10), realizado en 538 estudiantes de medicina, odontología y enfermería se encontró un 9,7\% de estudiantes con bajo peso, peso normal en $77,3 \%$ y sobrepeso y obesidad en 13\%. Por su parte, Poreba y Gać (2008) en Polonia, en un estudio con 240 estudiantes de la facultad de ciencias, reportan una proporción de sobrepeso de 7,7\% (11). Frecuencias similares a las de estudiantes de odontología en este estudio. Para algunos autores, los hábitos alimentarios de las personas con bajo peso se caracterizan por consumir menos de tres comidas al día, con diferencias estadísticamente significativas cuando se compara con los estudiantes de peso normal y sobrepeso $(10,12)$.

Los estudiantes de odontología tienen hábitos alimentarios irregulares en el número de comidas al día y en los horarios de alimentación, eventualmente, el ingreso al ambiente universitario ha inducido deterioro en su estilo de vida. Los horarios de las comidas son irregulares en un $67,6 \%$ en el desayuno, $47,9 \%$ en el almuerzo y $74,6 \%$ en la comida. Hallazgos similares demuestran que la nutrición de los estudiantes es irregular y difiere en los horarios y en el número de comidas; la mayoría de los estudiantes no siguen un régimen alimenticio y consumen la mayoría de productos durante la segunda mitad del día $(13,14)$.

La frecuencia de consumo de 4 comidas diarias en estudiantes argentinos (1) fue de $37.8 \%$ con horarios irregulares del $49.8 \%$, en estudiantes chinos (7) el consumo de 3 comidas al día fue de $79 \%$. Otro estudio que comparó estudiantes japoneses y coreanos reporta una irregularidad en el horario de 54.7\% (15). En estudiantes colombianos (4) se reportó un consumo de 3 comidas diarias del $42.7 \%$. En estudiantes polacos, se registraron hábitos nutricionales poco saludables, con un consumo irregular de comidas del 50\% (16). En contraste, Czapska y colaboradores (17) en un estudio de cohorte, encontraron que la mayoría de sus estudiantes consumen tres comidas al día. En el presente estudio, el número de comidas diarias consumidas disminuyó significativamente después del ingreso a la universidad (prueba t, $\mathrm{p}=0.03$ ).
Los tipos de alimentos que los estudiantes de odontología consumen en el desayuno son lácteos, huevos, cereales y frutas. Para el almuerzo, los estudiantes consumen carne de res, leguminosas, mezclas vegetales, tubérculos y plátanos, también consumen verduras, hortalizas y grasas. En la comida o cena, los estudiantes, por lo general, consumen los mismos alimentos que en el almuerzo excepto por las verduras, e incluyen lácteos y frutas.

El consumo de lácteos en el estudio hecho en Argentina fue $44.5 \%$ (1), vegetales y hortalizas en un $34.5 \%$, carne, huevos y leguminosas en un 37\%, para este estudio el consumo de carnes, huevos y leguminosas fue del $58.3 \%$. En el estudio de MacMilan (18) el consumo fue del 13.4\%. En estudiantes mexicanos fue de aproximadamente del $40 \%$ y en estudiantes españoles fue de 65\% (19). En estudiantes colombianos se reporta un consumo diario de leche del 53\%, y consumo de proteínas, principalmente de carne de res, en un $17.9 \%$ y huevos en un $15.4 \%$, se registró consumo de lácteos a la hora del desayuno en $64.3 \%(5)$

En esta investigación se reporta un consumo de frutas del 30.8\%. Skemiene L y colaboradores (10) en Lituania, reportan que sólo $20 \%$ de los estudiantes de medicina consumen diariamente $400 \mathrm{~g}$ de fruta como lo recomienda la OMS; destacan que los estudiantes consumen poca cantidad de pan, cereales, y pastas que constituyen la base de la pirámide nutricional. Antal y colaboradores reportan que el $32 \%$ de los estudiantes no consumen leche o derivados lácteos a diario, 36\% consumen frutas una vez al día o menos y sólo el $26 \%$ consumen pan integral o cereales a diario. $\mathrm{Al}$ momento de consumir refrigerios, los estudiantes de odontología incluyen principalmente lácteos $(49,2 \%)$, frutas $(47,6 \%)$ y grasas $(19,9 \%)$.

En este estudio el consumo de lácteos es del 64,3\%, otros estudios reportan consumos del 44.5\%, al 68\% $(1,5,14)$. El consumo de carnes, huevos y leguminosas fue del $58.3 \%$. Diferentes estudios hallan ingesta desde $13,4 \%$ hasta $65 \%$ en estudiantes universitarios españoles $(1,5,18,19)$. En esta investigación se reporta un consumo de frutas del 30.8\%. Skemiene y su equipo en Lituania, reportaron que sólo $20 \%$ de los estudiantes de medicina consumen diariamente $400 \mathrm{~g}$ de fruta como lo recomienda la OMS; destacan que los estudiantes consumen poca 
cantidad de pan, cereales, y pastas que constituyen la base de la pirámide nutricional (13). Antal y colaboradores reportan que el 36\% consumen frutas una vez al día o menos, y sólo el $26 \%$ consumen pan integral o cereales a diario (14). Al momento de consumir refrigerios, los estudiantes de odontología incluyen principalmente lácteos $(49,2 \%)$, frutas $(47,6 \%)$ y grasas $(19,9 \%)$.

Varios estudios reportan que los hábitos alimenticios de los estudiantes de ciencias de la salud no son saludables, de hecho, consumen elevadas cantidades de grasa, especialmente, de origen animal. Así mismo, consumen cantidades insuficientes de grasas vegetales, pescado, frutas y verduras, por lo tanto su alimentación puede estar carente de fibra soluble y vitaminas $(13,14)$. Otros autores destacan aspectos positivos en la nutrición de estudiantes como preferir la comida cocinada en casa, alta frecuencia de consumo de jugos de frutas y de agua mineral, y consumo esporádico de comidas rápidas y papas fritas $(5,16)$.

La mayoría de estudiantes ha percibido cambios en sus hábitos alimentarios después de ingresar a la universidad, esto obedece, principalmente, a la falta de tiempo, dificultades económicas y poca variedad de alimentos disponibles en la cafetería de la universidad. La mitad de estudiantes $(50,2 \%)$ han percibido disminución de peso, y se han mantenido en su peso $28,9 \%$. Los criterios de selección de los alimentos son principalmente por gusto $(57,2 \%)$, costo $(34,5 \%)$, calidad $(31 \%)$ y valor nutritivo $(18,5 \%)$.

Estas características de consumo se pueden relacionar con la inmediatez en la consecución de las comidas debido al poco tiempo disponible para alimentarse, el papel que juega ser aceptado por el grupo de amigos también puede influenciar esta decisión. Por esto, el valor nutritivo no tiene tanta importancia en la selección del alimento, lo que indica que el conocimiento acerca de una nutrición balanceada y la práctica son distantes, y están influenciados por el ambiente social del individuo. Las molestias asociadas a los cambios de hábitos son gastritis, cefalea y migraña, síntomas que estarían asociados a déficit en la nutrición y horarios irregulares de las comidas.

Para finalizar, es importante tener presente que una nutrición inadecuada y los estilos de vida poco saludables, son factores que favorecen el desarrollo de algunas enfermedades crónicas en la adultez (11). De manera que, los jóvenes inmersos en un sistema académico, con muchas exigencias físicas e intelectuales y formándose para el cuidado de la salud, requieren que las universidades revisen su ambiente de estudio con el ánimo de buscar coherencia entre el conocimiento y las prácticas cotidianas. $\mathrm{NO}$

\section{Referencias}

1. Horacio Gerometta P, Carrara C, Alberto Galarza L, Feyling V. Frecuencia de consumo de alimentos en ingresantes a la carrera de medicina: Rev posgrado de la VIa cátedra de medicina; 2004;136:913.

2. Alvarez Rosario J, Castaño Castrillón J, Marin Viatela J, Navas Galvis C, Noreña Vidal P, Ovalle Arciniegas H, et al. Estilos de vida en estudiantes de la universidad de Manizales, año 2006. Arch Med Manizales. 2007;7:46-56.

3. Sanabria P, González L, Urrego D. Estilos de vida saludable en profesionales de la salud colombianos estudio exploratorio. Rev Med. 2007;15:207-217.

4. Tobar Vargas L, Vásquez Cardoso S, Bautistas Muñoz L. Descripción de hábitos y comportamientos alimentarios de los estudiantes de la Facultad de Ciencias de la Pontificia Universidad Javeriana, 2005. Universitas Scientiarum. 2008;13:55-63.

5. Reia A, Cabrera J, Richard M. Calidad de vida y estado de salud de los estudiantes universitarios. Biblioteca virtual Miguel de Cervantes. 2001; 48-52.

6. World Health Organization. Obesity Preventing and Managing the Global Epidemic. Report of a WHO consultation. World Health Organ Tech Rep Ser 2000; 894: i-xii. 1-253.

7. Sakamaki R, Toyama K, Amamoto R, Liu CJ, Shinfuku N. Nutritional knowledge, food habits and health attitude of Chinese university students--a cross sectional study. Nutr J. 2005;4:4.

8. Sakamaki R, Amamoto R, MochidaY, Shinfuku N, Toyama K. A comparative study of food habits and body shape perception of university students in Japan and Korea. Nutr J. 2005;4:31.

9. Lowry R, Galuska DA, Fulton JE, Wechsler H, Kann L, Collins JL. Physical activity, food choice, and weight management goals and practices among US college students. Am J Prev Med. 2000;18:18-27.

10. Kolarzyk E, Kwiatkowski J, Lang-Młynarska D. Nutritional model and nutritional behaviors depending on BMI value among students of the Collegium Medicum of Jagiellonian University in Cracow. Przegl Lek. 2003;60 Suppl 6:43-47.

11. Poreba R, Gać P, Zawadzki M, Poreba M, Derkacz A, Pawlas K, Pilecki W, Andrzejak R. Life style and cardiovascular risk factors among students of Wroclaw postgraduate schools. Pol Arch Med Wewn. 2008;18:102-110.

12. Stefańska E, Ostrowska L, Czapska D, Karczewski J, Gołdowska E. Qualitative evaluation of the students diet with normal body mass and overweight at the Medical University in Bialystok. Przegl Lek. 2003;60:31-35.

13. Skemiene L, Ustinaviciene R, Piesine L, Radisauskas R. Peculiarities of medical students' nutrition. Medicina (Kaunas). 2007;43:145-152.

14. Antal M, Nagy K, Bíró L, Greiner E, Regöly-Mérei A, Domonkos A, Balajti A, Szabó C, Mozsáry E. Cross-sectional survey on the 
nutritional and life-style habits of secondary school students in Hungary] Orv Hetil. 2003;144:1631-1636.

15. Sakamaki R, Amamoto R, MochidaY, Shinfuku N, Toyama K. A comparative study of food habits and body shape perception of university students in Japan and Korea. Nutr J. 2005;4:31.

16. Uramowska-Zyto B, Kozłowska-Wojciechowska M, Jarosz A, Makarewicz-Wujec M. Dietary and life-style habits of university students in Poland--empirical study] Rocz Panstw Zakl Hig. 2004;55:171-179.
17. Czapska D, Ostrowska L, Stefańska E, Karczewski J. Chosen dietary habits in a cohort of students of the Medical University of Bialystok in the years 2000-2003. Rocz Panstw Zakl Hig. 2005;56:149-155.

18. MacMilan N. Valoración de Hábitos de alimentación, actividad física y condición nutricional en estudiantes de la pontificia universidad católica de Valparaíso. Rev Chil Nutr. 2007;34:330-336.

19. Díaz M, Riba M, Rodríguez A, Mora T. Patrón alimentario de estudiantes universitarios: Comparación entre culturas. Rev Esp Nutr Comunitaria. 2005;11: 8-11. 